\title{
Double Beta Decay: Historical Review of 75 Years of Research
}

\author{
A. S. Barabash ${ }^{1, *}$ \\ ${ }^{1}$ Institute of Theoretical and Experimental Physics, \\ B. Cheremushkinskaya 25, 117259 Moscow, Russia

\begin{abstract}
Main achievements during 75 years of research on double beta decay have been
\end{abstract} \\ reviewed. The existing experimental data have been presented and the capabilities \\ of the next-generation detectors have been demonstrated.
}

\section{INTRODUCTION}

The term "double beta decay" appeared 75 years ago. In view of this anniversary, I want to recall the main stages of the investigation of double beta $(2 \beta)$ decay and to review the main achievements in chronological order. In the course of time, many details (including important and interesting) are often forgotten, accents change, contributions of individual researchers are revised, etc. For this reason, young scientists beginning investigations of $2 \beta$ decay do not necessarily know earlier achievements, motivation, and persons responsible for certain achievements. I began to study $2 \beta$ decay in 1982 and is one of the "senior" (but not oldest!) researchers of $2 \beta$ decay continuing actively work in this field. Furthermore, I was happy to meet and work with almost all leading specialists in the field of $2 \beta$ decay. These are the reasons for this review.

The review is organized as follows. Section 2 is devoted to the appearance of the problem of $2 \beta$ decay, Section 3 presents information on first experiments on the search for this process, the history of achievements in the investigation of $2 \beta$ decay since 1960 is presented in Sections 4-6 in the chronological order. Each section ends with a brief list of the main achievements during the corresponding period and the list of collaborations and individual scientists significantly contributing to the advance in this field. Section 7 briefly presents the current status and prospects of the investigation of $2 \beta$ decay in the near future. The tables 4-6 at the end of the review summarizes the main (in my opinion) stages in the history

\footnotetext{
* E-mail: barabash@itep.ru
} 
of the investigation of $2 \beta$ decay. The contribution of Russian and Soviet scientists to the investigation of $2 \beta$ decay is discussed in the Conclusion.

When writing this review, I tried to be maximally unbiased and give weighed estimates of obtained results (taking into account the opinions of leading experts in the field of $2 \beta$ decay). Nevertheless, it is not clearly free of author's opinion and author's estimate of the importance of various experiments and contributions of individual scientists.

\section{2. "BIRTH" OF $2 \beta$ DECAY}

The problem of $2 \beta$ decay appears immediately after Pauli's hypothesis on the existence of neutrino in 1930 and the development of the theory of $\beta$ decay in 1933. This theory was developed by Italian physicist Fermi by analogy with quantum electrodynamics, but he introduced a new type of interactions, weak interaction, to describe $\beta$ decay. This theory provided good description of the $\beta^{-}$- and $\beta^{+}$decays of nuclei:

$$
\begin{aligned}
& (A, Z) \rightarrow(A, Z+1)+e^{-}+\bar{\nu}, \\
& (A, Z) \rightarrow(A, Z-1)+e^{+}+\nu,
\end{aligned}
$$

where $A$ is the atomic number, $Z$ is the charge number, $e^{-}$is the electron, $e^{+}$is the positron, $\nu$ is the neutrino, and $\bar{\nu}$ is the antineutrino. In 1935, Goeppert-Mayer [1] for the first time pointed to the possibility of the process of two-neutrino double beta $(2 \beta(2 \nu))$ decay , i.e., a process of the transformation of the $(A, Z)$ nucleus to the $(A, Z+2)$ nucleus with the emission of two electrons and two (anti)neutrinos:

$$
(A, Z) \rightarrow(A, Z+2)+2 e^{-}+2 \bar{\nu}
$$

It is interesting that Wigner proposed to consider such a possibility (as mentioned by Goeppert-Mayer [1]). In 1937, Majorana [2] theoretically showed that the conclusions of the theory of $\beta$ decay remain unchanged under the assumption of the existence of only one type of the neutrino having no antiparticle (i.e., $\nu \equiv \bar{\nu}$ ). Thus, the notion of the Majorana neutrino (in contrast to the Dirac neutrino) appeared, but the term Majorana neutrino appeared later. At the same year, Racah [3] was the first who pointed out that such a neutrino emitted, e.g., in process (1) can induce the reaction

$$
\nu+(A, Z) \rightarrow(A, Z+1)+e^{-}
$$


This reaction cannot be induced by a Dirac neutrino (i.e., $\nu \neq \bar{\nu}$ ), because an antineutrino is emitted in reaction (1), whereas a neutrino is absorbed in reaction (4). I agree with Pontecorvo [4] that Racah [3] did not consider neutrinoless double beta decay $(2 \beta(0 \nu))$, but analyzed the possibility of distinguishing Majorana and Dirac neutrinos in the processes of inverse $\beta$ decay using free neutrino fluxes (virtual intermediate state of a nucleus and virtual neutrino were not considered!). ${ }^{1}$ In 1938, Furry [5] analyzed the ideas by Majorana and Racah and arrived at rather pessimistic conclusions concerning experimental possibilities of distinguishing Majorana and Dirac neutrinos. These conclusions were primarily based on the absence of intense sources of neutrinos at that time (reactors were absent!). However, as early as in 1939, Furry [6] considered for the first time the $2 \beta(0 \nu)$ decay, i.e., the transformation of the $(A, Z)$ nucleus to the $(A, Z+2)$ nucleus accompanied by the emission of only two electrons. Furry introduced the following scheme for describing the $2 \beta(0 \nu)$ decay. The process occurs in two stages: (i) the initial $(A, Z)$ nucleus emits one electron and transits to a virtual intermediate state plus virtual $\bar{\nu}$ and (ii) this virtual $\bar{\nu}$ as $\nu$ (since $\nu \equiv \bar{\nu}$ ) is absorbed by the intermediate nucleus and induces its decay with the emission of the second electron. This process can be represented as follows:

$$
(A, Z) \rightarrow(A, Z+2)+2 e^{-}
$$

Thus, during five years (1935-1939), the main $2 \beta$ processes were proposed and the first estimates of the lifetimes of nuclei with respect to both $2 \nu$ - $\left(\sim 10^{21}-10^{22} \mathrm{yr}\right)$ and $0 \nu$ decay $\left(\sim 10^{15}-10^{16} \mathrm{yr}\right)$ were done. It was shown that investigation of these processes can provide valuable information on the properties of the neutrino. The main contribution was made by M. Goeppert-Mayer and W. Furry.

\section{FIRST EXPERIMENTS (THE 1940S-1950S)}

At that time, the main motivation for experiments on the search for $2 \beta$ decay was the possibility of determining the nature of the neutrino (who is right, Dirac or Majorana?). The theoretical estimates made at that time gave strongly different values for these two possibilities: $\left(0 \nu\right.$ decay) $\sim 10^{15}$ yr for the Majorana neutrino, whereas $(2 \nu$ decay $) \sim 10^{21} \mathrm{yr}$

\footnotetext{
${ }^{1}$ It is sometimes erroneously stated that Racah [3] was the first who proposed the idea of $2 \beta(0 \nu)$ decay.
} 
for the Dirac neutrino. It was clear that if the neutrino has the Majorana nature, the $2 \beta(0 \nu)$ decay could be detected by detectors existing at that time.

In view of the World War II, active scientific investigations resumed only in the second half of the 1940s. The first experiment on the search for the $2 \beta$ decay was conducted in 1948 by Fireman [7] who sought the $2 \beta$ decay of ${ }^{124} \mathrm{Sn}$ using Geiger counters and obtained the limit $T_{1 / 2}>3 \times 10^{15} \mathrm{yr}$. In 1949, Fireman carried out a new experiment with ${ }^{124} \mathrm{Sn}$ and obtained a positive result $T_{1 / 2}=(4-9) \times 10^{15} \mathrm{yr}[8]$. Already in that pioneering experiment, he used an enriched tin sample (25 g, enriched to $54 \%$ ). This result was not confirmed later in more sensitive experiments performed in 1951-1953 [9-13]. The best bound for ${ }^{124} \mathrm{Sn}$ was $T_{1 / 2}>2 \times 10^{17}$ yr [10]. In those years, "positive" results often appeared, but they disproved by later experiments. In particular, the "positive" effect was also observed in ${ }^{100} \mathrm{Mo}[14],{ }^{48} \mathrm{Ca}$ [15], and ${ }^{96} \mathrm{Zr}$ [13]. In those years, other $2 \beta$ decay processes with the emission of two positrons $\left(2 \beta^{+}\right)$and electron capture with the simultaneous emission of a positron $\left(\mathrm{EC} \beta^{+}\right)[14,16]$ were sought. Winter [17] also pointed for the first time to the possibility of a large increase in the probability of the neutrinoless two-electron capture $(\operatorname{ECEC}(0 \nu))$ to an excited level of the daughter nucleus under the resonance conditions (zero transition energy). To search for the $2 \beta$ processes, the most advanced methods and detectors were used. In particular, Geiger, proportional, and scintillation counters, Wilson chamber, and nuclear emulsion were used. Enriched isotopes $\left({ }^{48} \mathrm{Ca},{ }^{94} \mathrm{Zr},{ }^{96} \mathrm{Zr},{ }^{124} \mathrm{Sn}\right)$ were widely used. Instruments were placed deep under the ground (to suppress the background from cosmic rays); passive and active shieldings were used. Nevertheless, the sensitivity of counter experiments at that time was no more than $\sim 10^{17}-10^{18}$ yr. Note that the first experiment on the search for $2 \beta$ decay in the USSR was conducted in 1956 [18].

In 1949, the first geochemical experiment was performed with ${ }^{130} \mathrm{Te}$, which was transformed into ${ }^{130} \mathrm{Xe}$ after $2 \beta$ decay [19]. This method consists of the separation of xenon from ancient minerals (whose age is up to several billion years) and their subsequent isotope analysis. The detection of an excess amount of ${ }^{130} \mathrm{Xe}$ (taking into account the contributions to the effect from various nuclear reactions induced by neutrons, cosmic rays, etc.) indicates the $2 \beta$ decay of the initial nucleus and makes it possible to determine its half-life. The sensitivity of the first geochemical experiment was much higher than the sensitivities of counter experiments and a lower bound of $8 \times 10^{19} \mathrm{yr}$ was obtained. As early as in 1950, Inghram and Reynolds [20] detected the $2 \beta$ decay of ${ }^{130} \mathrm{Te}$ and obtained $T_{1 / 2}=1.4 \times 10^{21} \mathrm{yr}$. This 
result was initially not considered seriously, but it became clear after 15-20 yr that $2 \beta(2 \nu)$ decay was indeed observed for the first time in this experiment. This occurred as early as in 1950 !

The first radiochemical experiment with ${ }^{238} \mathrm{U}$ was also carried out in 1950 [21]. The aim of that experiment was to seek ${ }^{238} \mathrm{Pu}\left(T_{1 / 2}=87.7 \mathrm{yr}\right)$, which should appear after the $2 \beta$ decay of ${ }^{238} \mathrm{U}$. To this end, plutonium was separated from $14 \mathrm{~kg}$ of purified uranium oxide stored for six years and the presence of ${ }^{238} \mathrm{Pu}$ was detected by counting $\alpha$ particles. As a result, the limit $T_{1 / 2}>6 \times 10^{18}$ yr was obtained.

The main achievement of that period (as was understood many years later) was the first detection of $2 \beta(2 \nu)$ decay in the geochemical experiment with ${ }^{130}$ Te by Inghram and Reynolds.

\section{4. "MIDDLE AGES" (THE 1960S-1970S)}

After the discovery of space parity violation in 1957 and the determination of the $V-$ $A$ nature of weak interaction (this implies the presence of additional chiral selection), the probability of observing $2 \beta(0 \nu)$ decay decreased strongly, because it became clear that the probability of such a process can be much smaller than that for the $2 \beta(2 \nu)$ transition. Nevertheless, interest in the $2 \beta$ processes held in those years. In 1960, the probability of the $2 \beta(0 \nu)$ decay was calculated for the first time under the assumption of the existence of the Majorana mass of the neutrino [22]. Another possibility, namely, the admixture of right-handed currents under the additional assumption of the identity of the neutrino and antineutrino was simultaneously considered. At that time, it became clear that the search for $2 \beta(0 \nu)$ decay is a good test for the lepton number conservation law.

Experiments at that time were scarce but of a very high level. Mateosian and Goldhaber [23] achieved a sensitivity of $2 \times 10^{20} \mathrm{yr}$ in the experiments with ${ }^{48}$ Ca; i.e., a sensitivity of $10^{20} \mathrm{yr}$ was reached for the first time in counter experiments. The working substance of the detector was the enriched material under investigation (calcium fluoride crystal containing $11.4 \mathrm{~g}$ of ${ }^{48} \mathrm{Ca}$ enriched to $96.6 \%$ ). Thus, the "detector $\equiv$ studied substance" scheme was implemented for the first time. In 1967, Fiorini et al. [24] for the first time used a Ge(Li) detector to search for $2 \beta$ decay and immediately obtained the bound $T_{1 / 2}>3 \times 10^{20}$ yr on the $2 \beta(0 \nu)$ decay of ${ }^{76} \mathrm{Ge}$, which was the best result at that time for counter experiments. Finally, 
the bound $T_{1 / 2}>5 \times 10^{21}$ yr fantastic for that time was reached [25]. Correspondingly, wonderful prospects of the use of germanium semiconductor detectors to search for $2 \beta$ decay were demonstrated.

Almost at the same time, the research group led by Wu performed two remarkable experiments at a setup consisting of a streamer chamber in a magnetic field (visualization of tracks and measurement of the energy) plus plastic scintillators (simultaneity of the emission of electrons and the measurement of the energy of electrons). At this setup, very stringent bounds on $2 \beta(0 \nu)$ decay for isotopes with high energies of $2 \beta$ transitions were obtained: $T_{1 / 2}>2 \times 10^{21}$ yr for ${ }^{48} \mathrm{Ca}(4.272 \mathrm{MeV})[26,27]$ and $T_{1 / 2}>3.1 \times 10^{21}$ yr for ${ }^{82} \mathrm{Se}$ $(2.995 \mathrm{MeV})$ [28]. It is worth noting that the sensitivity of counter experiments for the first time exceeded $10^{21} \mathrm{yr}$ in 1967 in the experiment with ${ }^{48} \mathrm{Ca}$ [26].

It is worth noting that these results for ${ }^{48} \mathrm{Ca},{ }^{82} \mathrm{Se}$, and ${ }^{76} \mathrm{Ge}$ in the early 1980 s were considered as a lodestar. All those who began to study $2 \beta$ decay at that time compared their plans and dreams with those experiments. Significant efforts were required in order to reach such a sensitivity in new experiments. Even now (after 35-40 yr!), they continue to be delightful.

In the second half of the 1960s, several research groups carried out geochemical experiments with ${ }^{130} \mathrm{Te}$ and ${ }^{82} \mathrm{Se}$ : research groups led by Takaoka [64] and Kirsten [118] confirmed the result obtained in 1950 for ${ }^{130} \mathrm{Te}$ and the research group led by Kirsten observed the $2 \beta(2 \nu)$ decay of ${ }^{82} \mathrm{Se}[29]$. In 1975, the research group led by Manuel [30] observed for the first time the $2 \beta$ decay of ${ }^{128} \mathrm{Te}$ in the geochemical experiment and determined the ratio $T_{1 / 2}\left({ }^{130} \mathrm{Te}\right) / T_{1 / 2}\left({ }^{128} \mathrm{Te}\right)$.

The main achievements of that period are the sensitivity to $2 \beta(0 \nu)$ decay above $10^{21}$ yr in experiments with ${ }^{48} \mathrm{Ca},{ }^{76} \mathrm{Ge}$, and ${ }^{82} \mathrm{Se}$, the detection of $2 \beta(2 \nu)$ decay in geochemical experiments with ${ }^{130} \mathrm{Te},{ }^{128} \mathrm{Te}$, and ${ }^{82} \mathrm{Se}$. The main researchers were E. Fiorini, C. Wu, T. Kirsten, and O. Manuel.

\section{5. "RENAISSANCE" (THE 1980S-1990S)}

Interest in $2 \beta$ decay was renewed in the early 1980s in view of several circumstances. First, Lubimov et al. [31] stated the detection of a neutrino mass of $\sim 30 \mathrm{eV}$ in the experiment performed at the Institute for Theoretical and Experimental Physics to measure the $\beta$ 
spectrum of tritium (but this statement was not confirmed in the subsequent experiments). Second, the neutrino with a mass of several dozens eVs was considered as a candidate for dark matter in cosmology. Third, grand unified theories where the mass of the neutrino was naturally treated as the Majorana mass were formulated. The appearance of the Majorana mass means the breaking of the symmetry associated with lepton charge conservation. This circumstance again enhanced interest in the search for $2 \beta$ decay and initiated the activity of theorists and experimenters.

In 1981, the following new type of $2 \beta$ decay, decay with the emission of Majoron was considered [35]:

$$
(A, Z) \rightarrow(A, Z+2)+2 e^{-}+\chi^{0} .
$$

The Majoron $\chi^{0}$ is a massless Goldstone boson appearing under the global breakdown of the $B-L$ symmetry, where $B$ and $L$ are the baryon and lepton quantum numbers, respectively. If the Majoron exists, it can play a serious role in the history of the early Universe and in the evolution of stars.

In 1982, the Schechter-Valle theorem was formulated [36] according to which the observation of $2 \beta(0 \nu)$ decay will imply the existence of the Majorana mass of the neutrino in the framework of gauge theories. This statement became a serious theoretical reasons for experimenters to continue and improve their experiments.

In 1985, the fundamental theoretical work by Doi, Kotani, and Takasugi [37] appeared that remains an encyclopedia of the theory of $2 \beta$ decay at present. In that work, the energy and angular distributions of electrons were calculated with a good accuracy for the mass mechanism and right-handed currents and the possibility of distinguishing these mechanism in experiments using the features of the corresponding distributions was demonstrated.

The discrepancy between the theoretical predictions and experiment bounds for $2 \beta(0 \nu)$ decay was a serious problem for a long time. Theories usually predicted the decay rate one or two orders of magnitude higher than the existing experimental bounds. However, in 1986, Vogel and Zirnbauer [38] showed that the inclusion of the particle-particle interaction in a nucleus allows a fairly accurate calculation of the rate of $2 \nu$ decay in the framework of the quasiparticle random phase approximation (QRPA). For this reason, the QRPA models were widely used to calculate nuclear matrix elements for both $2 \nu$ - and $0 \nu$ decays.

The activity of experimenters increased strongly. Several dozens research groups began to 
search for $2 \beta$ decay. In the 1980 s, numerous measurements were performed with ${ }^{76}$ Ge using high purity germanium (HPGe) detectors, primarily because large HPGe detectors became available and quite cheap instruments. At the same time, the choice of low-background construction materials, the location of detectors surrounded by passive and active shieldings deep under the ground provided the significant reduction of the background (large contributions were made by Avignone, Brodzinski, and Fiorini). All these led to an increase in the sensitivity by several orders of magnitude. Several research groups soon obtained the limits of $10^{22}$ and $10^{23} \mathrm{yr}$. The best experimental limit $T_{1 / 2}>1.2 \times 10^{24} \mathrm{yr}$ on the $2 \beta(0 \nu)$ decay of ${ }^{76}$ Ge was obtained by Caldwell [39] with the $\sim 7.2-\mathrm{kg}$ high-purity natural Ge detectors. In 1987, semiconductor $\mathrm{Ge}(\mathrm{Li})$ detectors grown from enriched germanium were used for the first time (ITEP-ErPhI experiment [40, 41]). All these achievements allowed two large experiments with enriched germanium (Heidelberg-Moscow [42] and IGEX [43]), in which a sensitivity of $\sim 10^{25}$ yr to $2 \beta(0 \nu)$ decay was reached. A quiet stringent limit on $2 \beta(0 \nu)$ decay was also obtained for ${ }^{136} \mathrm{Xe}$ in the experiment with a time projection chamber with $3.3-\mathrm{kg}$ xenon enriched in ${ }^{136} \mathrm{Xe}$ to $62 \%$. In addition to the measurement of the total energy of electrons, events with the simultaneous emission of two electrons from one points were selected and tracks of electrons were reconstructed. After the measurements during almost a year, the limit $T_{1 / 2}>3.4 \times 10^{23}$ yr was obtained [44]. In 1984, Fiorini and Niinikoski [45] proposed using low-temperature detectors to search for $2 \beta$ decay and the Milano group successfully developed this method ${ }^{2}$.

The main experimental achievement in that period was the first observation of the $2 \beta(2 \nu)$ decay of ${ }^{82} \mathrm{Se}$ in the direct counter experiment with a time projection chamber by the research group led by Moe in 1987 [46]. Only $362 \beta$ events were detected and the value $T_{1 / 2}=1.1_{-0.3}^{+0.8} \times$ $10^{20} \mathrm{yr}$ was obtained. This result was very important, particularly from the psychological point of view. It removed a certain invisible barrier. Positive results for $2 \nu$ decay appeared as from a cornucopia. The $2 \beta(2 \nu)$ decay of ${ }^{76} \mathrm{Ge}[41],{ }^{100} \mathrm{Mo}[47,48],{ }^{116} \mathrm{Cd}[49-51]$, and other nuclei was soon detected (at that time, $2 \beta(2 \nu)$ decay was detected in direct experiments for seven nuclei). The $2 \beta(2 \nu)$ decay of most nuclei was observed in several independent experiments. The largest contribution was made by the research group led by Moe $\left({ }^{82} \mathrm{Se}[46\right.$,

\footnotetext{
${ }^{2}$ It is necessary to notice that for the first time this idea has been stated in JINR (Dubna) by Mizelmaher, Neganov and Trofimov in 1982 [119], but in JINR this idea was not realized.
} 
52], ${ }^{100} \mathrm{Mo}$ [48], ${ }^{150} \mathrm{Nd}$ [53], and ${ }^{48} \mathrm{Ca}[54]$ ), by Ejiri et al. ( ${ }^{100} \mathrm{Mo}$ [47] and ${ }^{116} \mathrm{Cd}$ [49]), and in a series of the experiments with the NEMO-2 detector $\left({ }^{100} \mathrm{Mo}[55],{ }^{116} \mathrm{Cd}[50,56],{ }^{82} \mathrm{Se}\right.$ [57], and ${ }^{96} \mathrm{Zr}$ [58]). Furthermore, the energy spectra and angular distributions of electrons for all investigated isotopes were studied in the NEMO-2 experiment. The $2 \beta(2 \nu)$ decay of ${ }^{238} \mathrm{U}$ was observed in a radiochemical experiment in 1991 [59]. In the same year, Barabash, Avignone, et al. detected for the first time $2 \beta(2 \nu)$ decay to the excited state of the daughter nucleus $\left({ }^{100} \mathrm{Mo}-{ }^{100} \mathrm{Ru}\left(0_{1}^{+} ; 1130.3 \mathrm{eV}\right)\right.$ transition; the final result was reported in [60]).

Geochemical experiments were actively conducted in the 1980s and almost stopped to the end of the 1990s. In the beginning of the 1980s, the half-lives of ${ }^{130} \mathrm{Te}$ and ${ }^{128} \mathrm{Te}$ were actively discussed. Several research groups [61, 62] reported large half-lives $\left(\sim 2.7 \times 10^{21}\right.$ and $\sim 7.7 \times 10^{24}$ yr, respectively), whereas other authors [63-65] obtained much smaller values $\left(\sim 0.8 \times 10^{21}\right.$ and $\sim 2 \times 10^{24} \mathrm{yr}$, respectively). Correspondingly, the results differ by a factor of $\sim 3.5$, although the stated measurement accuracy was quite high (the accuracy of some experiments reached 3\%, as, e.g., in [62]). This problem was not solved at that time. It was even assumed in [66] that this discrepancy was probably caused by the time dependence of the rate of $2 \beta$ decay (due, e.g., to time variations of the weak interaction constant), because small $T_{1 / 2}$ values were obtained for "young" minerals (ages of $\sim 10^{7}$ $10^{8} \mathrm{yr}$ ), whereas large values were obtained for "old" samples (ages of $\sim 10^{9} \mathrm{yr}$ or more). In 1993, the first geochemical experiment with ${ }^{96} \mathrm{Zr}$ was performed and the half-life for the ${ }^{96} \mathrm{Zr}-$ ${ }^{96} \mathrm{Mo}$ transition was obtained [67]. In this case, the daughter substance (molybdenum) is not a gas in contrast to xenon and krypton, which are the daughter elements in experiments with tellurium and selenium, respectively.

The main achievements in that period are the first observation of $2 \nu$ decay in a direct (counter) experiment, the achievement of sensitivity to $2 \beta(0 \nu)$ decay in ${ }^{76} \mathrm{Ge}$ higher than $10^{25} \mathrm{yr}$, the measurement of $T_{1 / 2}(2 \nu)$ for ten isotopes (in direct and geochemical experiments), and the first observation of $2 \beta(2 \nu)$ decay to the excited state of the daughter nucleus. The most contribution was made by the following researchers/collaborations: M. Moe, F. Avignone, A.S. Barabash, S. Jullian, H. Ejiri, Yu. G. Zdesenko, H. Klapdor-Kleingrothaus, and E. Fiorini/Heidelberg-Moscow, IGEX, and NEMO-2. The contribution of theorists S. Petcov, O. Civitarese, J. Suhonen, A. Faessler,and F. Simkovic (in addition to the researchers listed above) should be mentioned. 


\section{6. "CONTEMPORARY HISTORY" (FIRST DECADE OF THE 21ST CENTURY)}

In 1998, the observation of neutrino oscillations in an experiment with atmospheric neutrinos was reported at the international conference "Neutrino-98" (Takayama, Japan). More recently, neutrino oscillations were observed in experiments with solar, reactor, and accelerator neutrinos. This means that the neutrino has mass! In view of this circumstance, interest in $2 \beta(0 \nu)$ decay in the beginning of the 21st century increased strongly. It became clear that the detection and investigation of $2 \beta(0 \nu)$ decay can clarify many problems of neutrino physics:

(i) The nature (Dirac or Majorana?) of the neutrino mass.

(ii) The absolute scale of the neutrino mass (measurement or bound on the mass of the lightest neutrino).

(iii) Hierarchy type (normal, inverse, or quasidegenerate).

(iv) $C P$ violation in the lepton sector (measurement of the Majorana $C P$-violating phases).

At the very beginning of that period, the Heidelberg-Moscow Collaboration completed the measurements with ${ }^{76} \mathrm{Ge}$. The interpretation of the experimental results was ambiguous. First, the authors jointly reported the limit $T_{1 / 2}>1.9 \times 10^{25}$ yr [42]. Then, KlapdorKleingrothaus et al. stated the observation of $2 \beta(0 \nu)$ decay of ${ }^{76} \mathrm{Ge}$ with a half-life of $1.5 \times 10^{25}$ yr [68]. The Moscow part of the Collaboration disagreed with this statement [69]. Several years later, Klapdor-Kleingrothaus changed the half-life to $1.19 \times 10^{25}$ yr [70], and after two years, he again changed it to $2.23_{-0.31}^{+0.44} \times 10^{25}$ yr [71]. This "observation" was actively discussed and strongly criticized by many physicists [72-75], and it is now clear that only new experiments with ${ }^{76} \mathrm{Ge}$ can clarify the situation.

Important results concerning the search for $2 \beta(0 \nu)$ decay were obtained in the experiments CUORICINO [76,77,120] (completed in 2008) and NEMO-3 [78-84] (completed in January 2011). Those experiments involved large detectors with the masses of the studied substances of 40 and $10 \mathrm{~kg}$, respectively. The sensitivity to $2 \beta(0 \nu)$ decay reached in those experiments was higher than $10^{24} \mathrm{yr}\left(>2.8 \times 10^{24} \mathrm{yr}\right.$ for ${ }^{130} \mathrm{Te}[120]$ and $>1.1 \times 10^{24}$ yr for ${ }^{100} \mathrm{Mo}$ [83]). In view of the much larger phase-space volume of the studied nuclei $\left({ }^{130} \mathrm{Te}\right.$ and $\left.{ }^{100} \mathrm{Mo}\right)$, the sensitivity to the effective neutrino mass was comparable with the sensitivity in experiments with ${ }^{76}$ Ge. The NEMO-3 experiment simultaneously concerns seven different isotopes and 
$2 \beta(2 \nu)$ decay was detected for all these isotopes. In addition to the precision measurement of $T_{1 / 2}(2 \nu)$, this experiment includes the detection of all main characteristics of $2 \nu$ decay: the total energy spectrum, the energy spectrum of individual electrons and angular distribution. To the end of 2008, more than 700000 (!) $2 \nu$ events were detected for ${ }^{100}$ Mo against the almost zero background. The $T_{1 / 2}(2 \nu)$ value for ${ }^{130}$ Te was reliably measured for the first time and, thereby, the old dispute between geochemists was solved: the small current value of the half-life was confirmed. In the same years, interest in $2 \beta^{+}, \mathrm{EC} \beta^{+}$, and ECEC processes increased and special experiments were performed for the first time to seek the resonance ECEC transition to an excited state of the daughter nucleus (see review [85]).

Geochemical experiments were surprisingly revived at that time. Experiments with ${ }^{96} \mathrm{Zr}$ [86], ${ }^{100} \mathrm{Mo}[87],{ }^{130} \mathrm{Ba}[88]$, and ${ }^{130} \mathrm{Te}$ [89, 90] were performed. As a result, the $\mathrm{ECEC}(2 \nu)$ process in ${ }^{130} \mathrm{Ba}$ was observed for the first time [88] and a serious attempt was made to explain the existing discrepancies in the geochemical experiments with ${ }^{130} \mathrm{Te}[89,90]$. At the same time, the results for ${ }^{96} \mathrm{Zr}$ and ${ }^{100} \mathrm{Mo}$ are inconsistent with the results of the counter experiments.

The main achievements in that period are a controversial situation with the "positive" result for ${ }^{76} \mathrm{Ge}$ (can it be called an achievement?), new results for $2 \beta(0 \nu)$ decay in ${ }^{130} \mathrm{Te}$ and ${ }^{100} \mathrm{Mo}$, precision investigation of $2 \beta(2 \nu)$ decay for many nuclei, the first observation of the ECEC $(2 \nu)$ process. The main contribution was made by H. Klapdor-Kleingrothaus and Heidelberg-Moscow, CUORICINO, and NEMO-3 Collaborations.

\section{CURRENT STATUS AND PROSPECTS}

Tables 1-3 present the best current data on $2 \beta(2 \nu), 2 \beta(0 \nu)$ and $2 \beta\left(0 \nu \chi^{0}\right)$ decays. Tables 4-6 present the main historical landmarks of the 75 -yr investigations of these processes.

Thus, $2 \beta(2 \nu)$ decay has been detected for ten nuclei $\left({ }^{48} \mathrm{Ca},{ }^{76} \mathrm{Ge},{ }^{82} \mathrm{Se},{ }^{96} \mathrm{Zr},{ }^{100} \mathrm{Mo},{ }^{116} \mathrm{Cd}\right.$, ${ }^{128} \mathrm{Te},{ }^{130} \mathrm{Te},{ }^{150} \mathrm{Nd}$, and $\left.{ }^{238} \mathrm{U}\right)$. In addition, $2 \beta(2 \nu)$ decay of ${ }^{100} \mathrm{Mo}$ and ${ }^{150} \mathrm{Nd}$ to the $0_{1}^{+}$excited state of the daughter nuclei and $\operatorname{ECEC}(2 \nu)$ process in ${ }^{130} \mathrm{Ba}$ were detected. Experiments on $2 \beta(2 \nu)$ decay achieved a qualitatively new level, where not only the half-life, but also the other parameters of the process are measured with a high accuracy (NEMO-3 experiment). At the same time, the sensitivity of experiments on the search for $2 \beta$ decay with the transition to excited states of the daughter nuclei, as well as $2 \beta^{+}, \mathrm{EC} \beta^{+}$, and ECEC processes, increases 
continuously. As a result, the transition to thorough and comprehensive investigations of the $2 \nu$ processes is outlined, which provide very important information on the nuclear matrix elements, the parameters of various theoretical models, etc. A certain progress is observed in the calculations of the nuclear matrix elements and in the understanding of the nuclear physical aspects of $2 \beta$ decay, although the accuracy of the calculations of the nuclear matrix elements is still low.

Any $2 \beta(0 \nu)$ decay has not been detected and, according to Table 2, the conservative bound on the effective mass of the Majorana neutrino is $0.7 \mathrm{eV}$. The current conservative bound on the Majoron-neutrino coupling constant from the experiments on $2 \beta$ decay is $\left\langle g_{e e}\right\rangle<1.7 \times 10^{-4}$.

Experiments of the next generation with the mass of the studied isotopes $\sim 100-1000 \mathrm{~kg}$ will be launched in a few years and will provide a sensitivity of $0.1-0.01 \mathrm{eV}$ to the efficient mass of the Majorana neutrino, i.e., will allow the analysis of the region of the predictions of the scheme with the inverse neutrino-mass hierarchy. Table 7 presents the parameters of several most promising planned experiments. First phase of GERDA (18 kg of $\left.{ }^{76} \mathrm{Ge}\right)$, EXO-200 (200 kg of $\left.{ }^{136} \mathrm{Xe}\right)$, CUORE-0 ( $40 \mathrm{~kg}$ of natural Te) and KamLAND-Xe (400 kg of ${ }^{136} \mathrm{Xe}$ ) plan to start data-tacking in 2011. For this reason one can expect occurrance of new, very interesting results in 2011-2012.

\section{CONCLUSIONS}

To conclude, it is worth noting that a significant contribution to the study of the $2 \beta$ decay processes was made by Soviet/Russian physicists. The first Soviet theoretical work was published in 1950 by Sliv, who calculated the probability of $2 \beta$ decay [116]. In 1954, the first in world (of very high quality!) review of the investigations of $2 \beta$ decay was written by Zeldovich, Luk'yanov, and Smorodinsky [117]. From 1956 to 1965, Dobrokhotov, Lazarenko,

and Luk'yanov performed several high-quality (for that time) experiments with ${ }^{48} \mathrm{Ca}$ (as was mentioned in Section 3). In the 1980s, various Soviet research groups conducted numerous experiments and obtained world-level results (Baksan, Yerevan, Kiev, Moscow, Solotvino). At the end of the 1980s-beginning of the 1990s, active cooperation with foreign physicists began and joint experiments involving Russian scientists were conducted in almost all best underground laboratories of the world. One of the remarkable contributions of the Russian 
researchers is the use of enriched isotopes produced in the USSR/Russia. The largest experiments such as Heidelberg-Moscow, IGEX, NEMO-2, and NEMO-3 experiments would be impossible without Soviet/Russian scientists. At present, Russian physicists participate almost in all large projects of future experiments. Enriched isotopes (up to $1000 \mathrm{~kg}$ !) are planned to be produced in Russia. In addition to those mentioned above, I list only the leaders of Soviet/Russian research groups making noticeable contribution to the investigation of $2 \beta$ decay: A. S. Barabash, I. V. Kirpichnikov, and O. Ya. Zeldovich (Institute for Theoretical and Experimental Physics, Moscow), V. B. Brudanin (Joint Institute for Nuclear Research, Dubna, Moscow region), V. I. Lebedev (Russian Research Centre Kurchatov Institute, Moscow), Yu. G. Zdesenko (Institute for Nuclear Research, National Academy of Sciences of Ukraine, Kiev), and V. V. Kuzminov, A. A. Pomanskii, and A. A. Smolnikov (Institute for Nuclear Research, Russian Academy of Sciences, Moscow).

1. M. Goeppert-Mayer, Phys. Rev. 48, 512 (1935).

2. E. Majorana, Nuovo Cimento 14, 171 (1937).

3. G. Racah, Nuovo Cimento 14, 322 (1937).

4. B. M. Pontekorvo, Priroda, No. 1, 43 (1983).

5. W. H. Furry, Phys. Rev. 54, 56 (1938).

6. W. H. Furry, Phys. Rev. 56, 1184 (1939).

7. E. L. Fireman, Phys. Rev. 74, 1238 (1948).

8. E. L. Fireman, Phys. Rev. 75, 323 (1949).

9. J. S. Lowson, Phys. Rev. 81, 299 (1951).

10. M. I. Kalkstein, and W. F. S. Libby, Phys. Rev. 85, 368 (1952).

11. R. W. Pearce and E. R. Darby, Phys. Rev. 86, 1049 (1952).

12. E. L. Fireman, Phys. Rev. 86, 451 (1952).

13. J. A. McCarthy, Phys. Rev. 90, 853 (1953).

14. J. H. Fremlin and M. C. Walters, Proc. Phys. Soc. London, Sect. A 65, 911 (1952).

15. J. A. McCarthy, Phys. Rev. 97, 1234 (1955). 
16. A. Berthelot et al., Compt. Rend. 236, 1769 (1953).

17. R. G. Winter, Phys. Rev. 99, 88 (1955).

18. E. N. Dobrokhotov, V. R. Lazarenko, and S. Yu. Luk'yanov, Dokl. Akad. Nauk SSSR 110, 966 (1956) [Sov. Phys. Dokl. 1, 600 (1956)].

19. M. G. Inghram, and J. H. Reynolds, Phys. Rev. 76, 1265 (1949).

20. M. G. Inghram, and J. H. Reynolds, Phys. Rev. 78, 822 (1950).

21. C. B. Levine, A. Griorso, and G. T. Seaborg, Phys. Rev. 77, 296 (1950).

22. E. Greuling and R. C. Whitten, Ann. Phys (N.Y.). 11, 510 (1960).

23. E. der Mateosian and M. Goldhaber, Phys. Rev. 146, 810 (1966).

24. E. Fiorini et al., Phys. Lett. B 25, 602 (1967).

25. E. Fiorini et al., Nuovo Cimento A 13, 747 (1973).

26. R. K. Bardin, P. J. Gollon, J. D. Ullman, and C. S. Wu, Phys. Lett. B 26, 112 (1967).

27. R. K. Bardin, P. J. Gollon, J. D. Ullman, and C. S. Wu, Nucl. Phys. A 158, 337 (1970).

28. B. T. Cleveland, W. R. Leo, C. S. Wu, et al., Phys. Rev. Lett. 35, 757 (1975).

29. T. Kirsten, W. Gentner, and O. A. Schaeffer, Z. Phys. 202, 273 (1967).

30. E. W. Hennecke, O. K. Manuel, and D. D. Sabu, Phys. Rev. C 11, 1378 (1975).

31. V. A. Lubimov et al., Phys. Lett. B 94, 266 (1980).

32. Y. Chikashige, R. N. Mohapatra, and R. D. Peccei, Phys. Lett. B 98, 265 (1981).

33. C. S. Aulakh and R. N. Mohapatra, Phys. Lett. B 119, 136 (1982).

34. G. B. Gelmini and M. Roncadelli, Phys. Lett. B 99, 411 (1981).

35. H. M. Georgi, S. L. Glashow, and S. Nussinov, Nucl. Phys. B 193, 297 (1981).

36. J. Schechter and J. W. F. Valle, Phys. Rev. D 25, 2951 (1982).

37. M. Doi, T. Kotani, and E. Takasugi, Prog. Theor. Phys. Suppl. 83, 1 (1985).

38. P. Vogel and M. R. Zirnbauer, Phys. Rev. Lett. 57, 3148 (1986).

39. D. O. Caldwell, J. Phys. G 17, S137 (1991).

40. A. A. Vasenko et al., in Proceedings of the 2nd International Symposium on Underground Physics'87, Baksan Valley, USSR, Aug. 17-19, 1987 (Nauka, Moscow, 1988), p. 288. 
41. A. A. Vasenko et al., Mod. Phys. Lett. A 5, 1299 (1990).

42. H. V. Klapdor-Kleingrothaus et al., Eur. Phys. J. A 12, 147 (2001).

43. C. E. Aalseth et al., Phys. Rev. C 65, 09007 (2002).

44. R. Luescher et al., Phys. Lett. B 434, 407 (1998).

45. E. Fiorini and T. O. Niinikoski, Nucl. Instrum. Methods Phys. Res. 224, 83 (1984).

46. S. R. Elliott, A. A. Hahn, and M. K. Moe, Phys. Rev. Lett. 59, 2020 (1987).

47. H. Ejiri et al., Phys. Lett. B 258, 17 (1991).

48. S. R. Elliott, M. K. Moe, M. A. Nelson, and M. A. Vient, J. Phys. G 17, S145 (1991).

49. H. Ejiri et al., J. Phys. Soc. Jpn 64, 339 (1995).

50. R. Arnold et al., JETP Lett. 61, 170 (1995).

51. F. A. Danevich et al., Phys. Lett. B 344, 72 (1995).

52. S. R. Elliott et al., Phys. Rev. C 46, 1535 (1992).

53. A. De Silva et al., Phys. Rev. C 56, 2451 (1997).

54. A. Balysh et al., Phys. Rev. Lett. 77, 5186 (1996).

55. D. Dassié et al., Phys. Rev. D 51, 2090 (1995).

56. R. Arnold et al., Z. Phys. C 72, 239 (1996).

57. R. Arnold et al., Nucl. Phys. A 636, 209 (1998).

58. R. Arnold et al., Nucl. Phys. A 658, 299 (1999).

59. A. L. Turkevich, T. E. Economou, and G. A. Cowan, Phys. Rev. Lett. 67, 3211 (1991)

60. A. S. Barabash et al., Phys. Lett. B 345, 408 (1995).

61. T. Kirsten et al., in Proceedings of the International Symposium on Nuclear Beta Decay and Neutrino (Osaka'86) (World Sci., Singapore, 1986), p. 81.

62. T. Bernatowicz et al., Phys. Rev. C 47, 806 (1993).

63. O. K. Manuel, in Proceedings of the International Symposium on Nuclear Beta Decay and Neutrino (Osaka'86) (World Sci., Singapore, 1986), p. 71.

64. N. Takaoka, and K. Ogata, Z. Naturforsch. A 21, 84 (1966).

65. N. Takaoka, Y. Motomura, and K. Nagano, Phys. Rev. C 53, 1557 (1996). 
66. A. S. Barabash, JETP Lett., 68, 1 (1998); Eur. Phys. J. A 8, 137 (2000); Astrophys. Space Sci. 283, 607 (2003).

67. A. Kawashima, K. Takahashi, and A. Masuda, Phys. Rev. C 47, 2452 (1993).

68. H. V. Klapdor-Kleingrothaus et al., Mod. Phys. Lett. A 16, 2409 (2001).

69. A. M. Bakalyarov et al., Phys. Part. Nucl. Lett. 2, 77 (2005); hep-ex/0309016.

70. H. V. Klapdor-Kleingrothaus, I. V. Krivosheina, A. Dietz, and O. Chkvorets, Phys. Lett. B 586, 198 (2004).

71. H. V. Klapdor-Kleingrothaus and I. V. Krivosheina, Mod. Phys. Lett. A 21, 1547 (2006).

72. C. E. Aalseth et al., Mod. Phys. Lett. A 17, 1475 (2002).

73. Yu. G. Zdesenko, F. A. Danevich, and V. I. Treyak, Phys. Lett. B 546, 206 (2002).

74. A. Strumia and F. Vissani, Nucl. Phys. B 726, 294 (2005).

75. O. Chkvorets, arXiv:0812.1206 [hep-ex].

76. C. Arnaboldi et al., Phys. Rev. Lett. 95, 142501 (2005).

77. C. Arnaboldi et al., Phys. Rev. C 78, 035502 (2008).

78. R. Arnold et al., Nucl. Instrum. Methods. A 536, 79 (2005).

79. R. Arnold et al., Phys. Rev. Lett. 95, 182302 (2005).

80. R. Arnold et al., Nucl. Phys. A 765, 483 (2006).

81. R. Arnold et al., Nucl. Phys. A 781, 209 (2007).

82. J. Argyriades et al., Phys. Rev. C 80, 032501(R) (2009).

83. A. S. Barabash and V. B. Brudanin, Phys. At. Nucl. 74, 312 (2011).

84. J. Argyriades et al., Nucl. Phys. A 847, 168 (2010).

85. A. S. Barabash, Yad. Fiz. 73, 166 (2010) [Phys. At. Nucl. 73, 162 (2010)]; arXiv:0807.2948 [hep-ex].

86. M. E. Wieser and J. R. De Laeter, Phys. Rev. C 64, 024308 (2001).

87. H. Hidaka, C. V. Ly, and K. Suzuki, Phys. Rev. C 70, 025501 (2004).

88. A. P. Meshik, C.M. Hohenberg, O.V. Pravdivtseva, and Y.S. Kapusta, Phys. Rev. C 64, 035205 (2001).

89. A. P. Meshik, C.M. Hohenberg, O.V. Pravdivtseva, et al., Nucl. Phys. A 809, 275 (2008). 
90. H. V. Tomas et al., Phys. Rev. C 78, 054606 (2008).

91. A. S. Barabash, Phys. Rev. C 81, 035501 (2010).

92. M. Kortelainen and J. Suhonen, Phys. Rev. C 75, 051303(R) (2007).

93. M. Kortelainen and J. Suhonen, Phys. Rev. C 76, 024315 (2007).

94. F. Simkovic et al., Phys. Rev. C 77, 045503 (2008).

95. E. Caurier et al., Phys. Rev. Lett. 100, 052503 (2008).

96. J. Barea and F. Iachello, Phys. Rev. C 79, 044301 (2009).

97. K. Chaturvedi et al., Phys. Rev. C 78, 054302 (2008).

98. J. Suhonen and O. Civitarese, Phys. Rep. 300, 123 (1998).

99. S. Umehara et al., Phys. Rev. C 78, 058501 (2008).

100. F. A. Danevich et al., Phys. Rev. C 68, 035501 (2003).

101. R. Bernabei et al., Phys. Lett. B 546, 23 (2002).

102. A. S. Barabash, Phys. Lett. B 216, 257 (1989).

103. C. Arnaboldi et al., Phys. Lett. B 557, 167 (2003).

104. C. Arnaboldi et al., Nucl. Instrum. Methods Phys. Res. A 518, 775 (2004).

105. I. C. Bandac, J. Phys. Conf. Ser. 110, 082001 (2008).

106. I. Abt et al., hep-ex/0404039.

107. J. Janicsko-Csathy, Nucl. Phys. B Proc. Suppl. 188, 68 (2009).

108. R. Gaitskell et al., Majorana White Paper, nucl-ex/0311013.

109. V. E. Guiseppe, arXiv:0811.2446 [nucl-ex].

110. M. Danilov et al., Phys. Lett. B 480, 12 (2000).

111. R. Gornea, J. Phys. Conf. Ser. 179, 012004 (2009).

112. A. S. Barabash, Czech. J. Phys. 52, 575 (2002).

113. A. S. Barabash, Yad. Fiz. 67, 2008 (2004) [Phys. At. Nucl. 67, 1984 (2004)].

114. E. Chauveau, AIP Conf. Proc. 1180, 26 (2009).

115. K. Nakamura, Report at International Conference Neutrino'2010, Athens, Greece, 13-19 June, 2010. 
116. L. A. Sliv, Zh. Eksp. Teor. Fiz. 20, 1035 (1950).

117. Ya. B. Zeldovich, S. Yu. Luk'yanov, and Ya. A. Smorodinsky, Usp. Fiz. Nauk 54, 361 (1954).

118. T. Kirsten, W. Gentner, and O. Muller, Z. Naturforsch. 22a, 1783 (1967).

119. G. V. Mizelmaher, B. S. Neganov, V. N. Trofimov, Communication of JINR P8-82-549, Dubna, 1982 (in Russian).

120. E. Andreotti et al., Astropart. Phys. 34, 822 (2011).

121. J. Maneira (SNO+ Collabotion), Neutrino Oscillation Workshop (Conca Soecchiulla, Italy, September 4-11, 2010). 
Table 1. Current $T_{1 / 2}(2 \nu)$ values (taken from [91])

\begin{tabular}{l|r}
\hline \multicolumn{1}{c|}{ Isotope } & \multicolumn{1}{c}{$T_{1 / 2}(2 \nu), \mathrm{yr}$} \\
\hline${ }^{48} \mathrm{Ca}$ & $4.4_{-0.5}^{+0.6} \times 10^{19}$ \\
${ }^{76} \mathrm{Ge}$ & $(1.5 \pm 0.1) \times 10^{21}$ \\
${ }^{82} \mathrm{Se}$ & $(0.92 \pm 0.07) \times 10^{20}$ \\
${ }^{96} \mathrm{Zr}$ & $(2.3 \pm 0.2) \times 10^{19}$ \\
${ }^{100} \mathrm{Mo}$ & $(7.1 \pm 0.4) \times 10^{18}$ \\
${ }^{100} \mathrm{Mo}-{ }^{100} \mathrm{Ru}\left(0_{1}^{+}\right)$ & $5.9_{-0.6}^{+0.8} \times 10^{20}$ \\
${ }^{116} \mathrm{Cd}$ & $(2.8 \pm 0.2) \times 10^{19}$ \\
${ }^{128} \mathrm{Te}$ & $(1.9 \pm 0.4) \times 10^{24}$ \\
${ }^{130} \mathrm{Te}$ & $6.8_{-1.1}^{+1.2} \times 10^{20}$ \\
${ }^{150} \mathrm{Nd}$ & $(8.2 \pm 0.9) \times 10^{18}$ \\
${ }^{150} \mathrm{Nd}-{ }^{150} \mathrm{Sm}\left(0_{1}^{+}\right)$ & $1.33_{-0.26}^{+0.45} \times 10^{20}$ \\
${ }^{238} \mathrm{U}$ & $(2.0 \pm 0.6) \times 10^{21}$ \\
${ }^{130} \mathrm{Ba}, \mathrm{ECEC}(2 \nu)$ & $(2.2 \pm 0.5) \times 10^{21}$ \\
\hline
\end{tabular}


Table 2. Best current results concerning the search for $2 \beta(0 \nu)$ decay

\begin{tabular}{l|l|l|c}
\hline Isotope & $E_{2 \beta}, \mathrm{keV}$ & $T_{1 / 2}, \mathrm{yr}$ & $\left\langle m_{\nu}\right\rangle, \mathrm{eV}$ \\
\hline${ }^{48} \mathrm{Ca}$ & 4272 & $>5.8 \times 10^{22}[99]$ & $<14$ \\
${ }^{76} \mathrm{Ge}$ & 2039.0 & $>1.9 \times 10^{25}[42]$ & $<0.22-0.66$ \\
${ }^{82} \mathrm{Se}$ & 2996 & $>3.6 \times 10^{23}[83]$ & $<0.89-2.4$ \\
${ }^{96} \mathrm{Zr}$ & 3350 & $>9.2 \times 10^{21}[84]$ & $<7.2-19.5$ \\
${ }^{100} \mathrm{Mo}$ & 3034.4 & $>1.1 \times 10^{24}[83]$ & $<0.45-0.93$ \\
${ }^{116} \mathrm{Cd}$ & 2805 & $>1.7 \times 10^{23}[100]$ & $<1.2-2.7$ \\
${ }^{128} \mathrm{Te}$ & 867 & $>1.5 \times 10^{24}$ (geochemistry) [63, 91] & $<1.7-4.3$ \\
${ }^{130} \mathrm{Te}$ & 2527.5 & $>2.8 \times 10^{24}[120]$ & $<0.35-0.77$ \\
${ }^{136} \mathrm{Xe}$ & 2458.7 & $>4.5 \times 10^{23}[101]$ & $<1.1-2.7$ \\
${ }^{150} \mathrm{Nd}$ & 3367 & $>1.8 \times 10^{22}[82]$ & $<4.8-7.6$ \\
\hline
\end{tabular}

Note. All bounds are given with $90 \%$ C.L. The bounds on the effective mass of the Majorana neutrino $\left\langle m_{\nu}\right\rangle$ were obtained using the calculated nuclear matrix elements from [92-97] and phase-space volumes from [98]. A decrease in the nuclear matrix elements for ${ }^{150} \mathrm{Nd}$ caused by the deformation of the nucleus is taken into account (see [82]). 
Table 3. Best results concerning the search for $2 \beta\left(0 \nu \chi^{0}\right)$ decay ("odinary" Majoron)

\begin{tabular}{l|l|r}
\hline isotope & \multicolumn{1}{|c|}{$T_{1 / 2}$, yr } & \multicolumn{1}{|c}{$\left\langle g_{e e}\right\rangle$} \\
\hline${ }^{48} \mathrm{Ca}$ & $>7.2 \times 10^{20}[102]$ & $<1.4 \times 10^{-3}$ \\
${ }^{76} \mathrm{Ge}$ & $>6.4 \times 10^{22}[42]$ & $<(0.79-2.3) \times 10^{-4}$ \\
${ }^{82} \mathrm{Se}$ & $>1.5 \times 10^{22}[80]$ & $<(0.64-1.7) \times 10^{-4}$ \\
${ }^{96} \mathrm{Zr}$ & $>1.9 \times 10^{21}[84]$ & $<(1.5-5.7) \times 10^{-4}$ \\
${ }^{100} \mathrm{Mo}$ & $>2.7 \times 10^{22}[80]$ & $<(0.41-0.84) \times 10^{-4}$ \\
${ }^{116} \mathrm{Cd}$ & $>8 \times 10^{21}[100]$ & $<(0.81-1.9) \times 10^{-4}$ \\
${ }^{128} \mathrm{Te}$ & $>1.5 \times 10^{24}$ (geochemistry) [63, 91] & $<(0.67-1.7) \times 10^{-4}$ \\
${ }^{130} \mathrm{Te}$ & $>2.2 \times 10^{21}[103]$ & $<(1.6-4.3) \times 10^{-4}$ \\
${ }^{136} \mathrm{Xe}$ & $>1.6 \times 10^{22}[101]$ & $<(0.87-2.4) \times 10^{-4}$ \\
${ }^{150} \mathrm{Nd}$ & $>1.5 \times 10^{21}[82]$ & $<(1.7-3) \times 10^{-4}$ \\
\hline
\end{tabular}

Note. All bounds are given with $90 \%$ C.L. The bounds on the Majoron-neutrino coupling constant $\left\langle g_{e e}\right\rangle$ were obtained using the calculated nuclear matrix elements from [92-97] and phase-space volumes from [98]. A decrease in the nuclear matrix elements for ${ }^{150} \mathrm{Nd}$ caused by the deformation of the nucleus is taken into account (see [82]). 
Table 4. Main "landmarks" in double beta decay search.

\begin{tabular}{|c|c|c|}
\hline Date & Event & Remarks \\
\hline 1935 & Idea of $2 \beta 2(\nu)$ decay & M. Goeppert-Mayer [1] \\
\hline 1939 & Idea of $2 \beta 0(\nu)$ decay & W.H. Furry [6] \\
\hline 1948 & $\begin{array}{l}\text { First experiment for search for } 2 \beta \\
\text { decay }\end{array}$ & $\begin{array}{l}\text { E.L. Fireman }[7,8] ; \text { (Geiger counters and } 25 \mathrm{~g} \text { of } \\
\text { enriched }{ }^{124} \text { Sn were used) }\end{array}$ \\
\hline 1950 & $\begin{array}{l}\text { The first observation of } \\
\qquad 2 \beta 2(\nu) \text { decay }\end{array}$ & $\begin{array}{l}\text { M.G. Inghram, and J.H. Reynolds [20] } \\
\text { (geochem. experiment with }{ }^{130} \mathbf{T e} \text { ); } \\
\qquad T_{1 / 2} \approx 1.4 \cdot 10^{21} \mathbf{y}\end{array}$ \\
\hline 1966 & $\begin{array}{c}\text { First counter experiment } \\
\text { with sensitivity higher than } 10^{20} \mathrm{y}\end{array}$ & 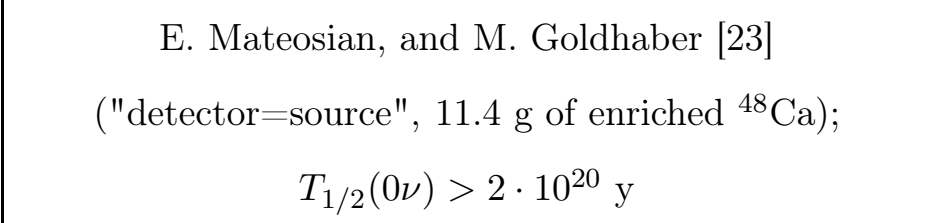 \\
\hline 1967 & $\begin{array}{l}\text { First experiment with a Ge } \\
\text { semiconductor detector }\end{array}$ & $\begin{array}{l}\text { E. Fiorini et al. [24] }\left(17 \mathrm{~cm}^{3} \mathrm{Ge}(\mathrm{Li}) \text { detector }\right. \\
\text { at the see level }) ; T_{1 / 2}(0 \nu)>3 \cdot 10^{20} \mathrm{y}\end{array}$ \\
\hline 1967 & $\begin{array}{l}\text { Observation of } 2 \beta(2 \nu) \text { decay } \\
\text { of }{ }^{82} \mathrm{Se}\end{array}$ & $\begin{array}{l}\text { T. Kirsten et al. [29] (geochemical experiment); } \\
\qquad T_{1 / 2} \approx 0.6 \cdot 10^{20} \mathrm{y}\end{array}$ \\
\hline $1967-$ & First counter experiment with & R.K. Bardin, P.J. Gollon, J.D. Ullman, and \\
\hline 1970 & sensitivity of higher than $10^{21} \mathrm{y}$ & $\begin{array}{c}\text { C.S. Wu }[26,27] \text { (strimmer chamber }+ \\
\text { scintillation counters }) ; T_{1 / 2}\left(0 \nu ;{ }^{48} \mathrm{Ca}\right)>2 \cdot 10^{21} \mathrm{y} \\
T_{1 / 2}\left(2 \nu ;{ }^{48} \mathrm{Ca}\right)>3.6 \cdot 10^{19} \mathrm{y}\end{array}$ \\
\hline 1973 & $\begin{array}{l}\text { High-sensitive counter experiment } \\
\text { with }{ }^{76} \mathrm{Ge}\end{array}$ & $\begin{array}{l}\text { E. Fiorini et al. [25] }\left(68 \mathrm{~cm}^{3} \mathrm{Ge}(\mathrm{Li}) \text { detector }\right. \\
\text { at } 4200 \mathrm{~m} \text { w.e. depth }) ; T_{1 / 2}(0 \nu)>5 \cdot 10^{21} \mathrm{y}\end{array}$ \\
\hline 1975 & $\begin{array}{c}\text { High-sensitive counter experiment } \\
\text { with }{ }^{82} \mathrm{Se}\end{array}$ & $\begin{array}{l}\text { B.T. Cleveland et al. [28] (streamer chamber }+ \\
\text { scint. counters); } T_{1 / 2}\left(0 \nu ;{ }^{82} \mathrm{Se}\right)>3.1 \cdot 10^{21} \mathrm{y}\end{array}$ \\
\hline $\begin{array}{l}1980- \\
1981\end{array}$ & $\begin{array}{c}\text { Idea of } 2 \beta \text { decay with Majoron } \\
\text { emission }\end{array}$ & $\begin{array}{l}\text { Models with singlet [32], doublet [33] and } \\
\text { triplet }[34,35] \text { Majoron were consided }\end{array}$ \\
\hline 1982 & Schechter-Valle theorem & $\begin{array}{l}\text { J. Schechter, and J.W.F. Valle [36]; in gauge theories } \\
\text { the detection of } 2 \beta(0 \nu) \text { decay means that the neutrinos } \\
\text { has a mass and this mass is of the Majorana type }\end{array}$ \\
\hline
\end{tabular}


Table 5. Main "landmarks" in double beta decay search (continuation of Table 4).

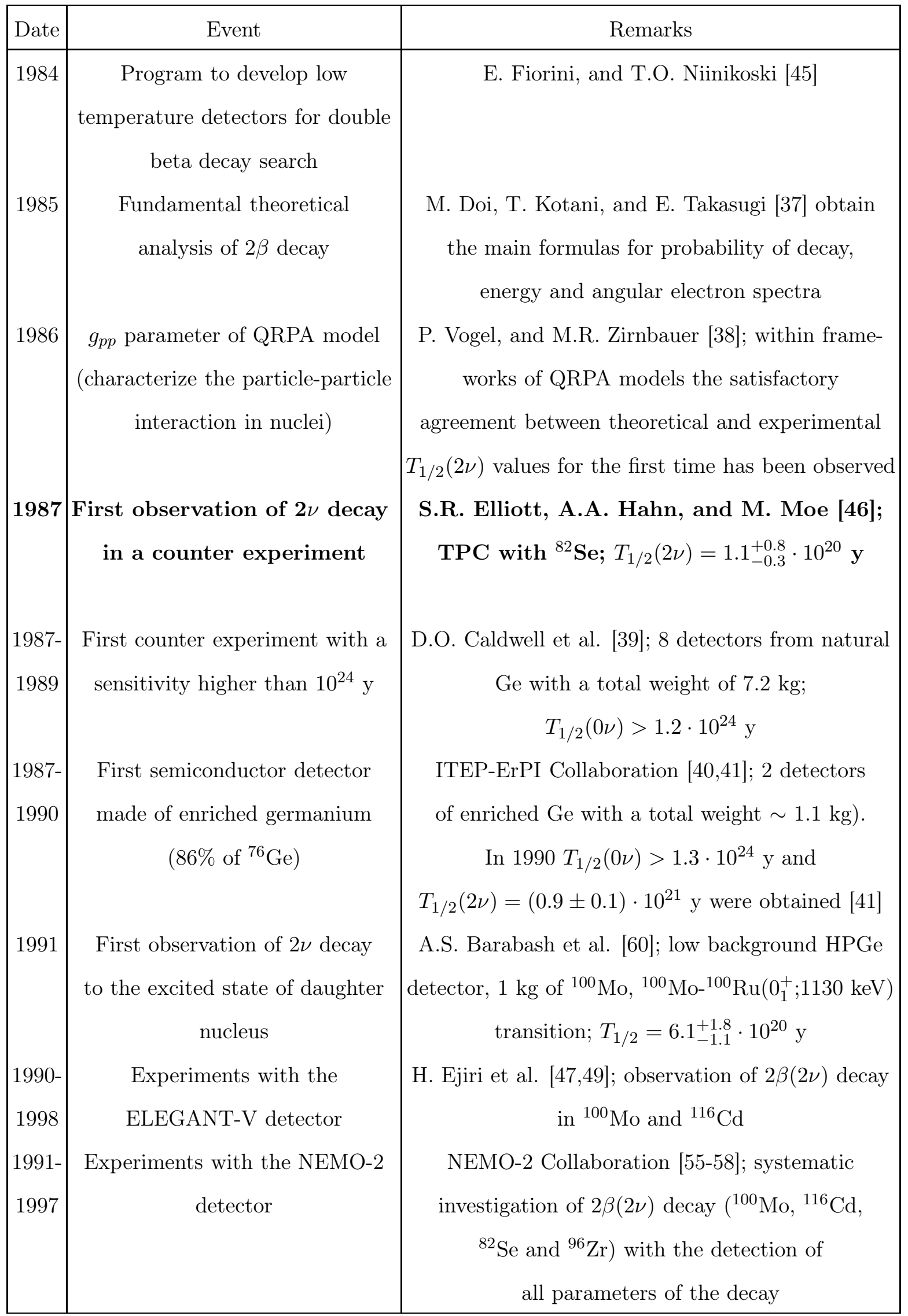


Table 6. Main "landmarks" in double beta decay search (continuation of Table 5).

\begin{tabular}{|c|c|c|}
\hline Date & Event & Remarks \\
\hline $\begin{array}{l}1991- \\
1999\end{array}$ & IGEX experiment & $\begin{array}{l}\text { Measurements with } 6.5 \mathrm{~kg} \text { of enriched }{ }^{76} \mathrm{Ge} ; \\
\qquad T_{1 / 2}(0 \nu)>1.57 \cdot 10^{25} \text { y }[43]\end{array}$ \\
\hline $1990-$ & Heidelberg-Moscow & Measurements with $11 \mathrm{~kg}$ of enriched ${ }^{76} \mathrm{Ge}$ [42]; \\
\hline 2003 & experiment & $\begin{array}{c}T_{1 / 2}(0 \nu)>1.9 \cdot 10^{25} \mathrm{y} \\
T_{1 / 2}(2 \nu)=1.74 \pm 0.01(\text { stat })_{-0.16}^{+0.18}(\text { syst }) \cdot 10^{21} \mathrm{y}\end{array}$ \\
\hline 2001 & First observation of $\operatorname{ECEC}(2 \nu)$ & $\begin{array}{c}\text { Geochemical experiment with }{ }^{130} \mathrm{Ba} \\
T_{1 / 2}=(2.2 \pm 0.5) \cdot 10^{21} \text { y }[88]\end{array}$ \\
\hline $2002-$ & NEMO-3 experiment & NEMO-3 Collaboration [78-84]; \\
\hline 2010 & & $\begin{array}{c}T_{1 / 2}\left(0 \nu ;{ }^{100} \mathrm{Mo}\right)>1.1 \cdot 10^{24} \mathrm{y} \text {; observation and } \\
\text { precise investigation of } 2 \beta(2 \nu) \text { decay for } 7 \text { isotopes } \\
\quad\left({ }^{48} \mathrm{Ca},{ }^{82} \mathrm{Se},{ }^{96} \mathrm{Zr},{ }^{100} \mathrm{Mo},{ }^{116} \mathrm{Cd},{ }^{130} \mathrm{Te},{ }^{150} \mathrm{Nd}\right)\end{array}$ \\
\hline $2003-$ & CUORICINO experiment & $\begin{array}{l}\text { CUORICINO Collaboration }[76,77,120] \\
\qquad T_{1 / 2}\left(0 \nu ;{ }^{130} \mathrm{Te}\right)>2.8 \cdot 10^{24} \mathrm{y}\end{array}$ \\
\hline
\end{tabular}


Table 7. Seven most developed and promising projects. Sensitivity at $90 \%$ C.L. for three (1-st steps of GERDA and MAJORANA, KamLAND, SNO+), five (EXO, SuperNEMO and CUORE), and ten (full-scale GERDA and MAJORANA) years of measurements is presented. *) For the background 0.001 $\mathrm{keV}^{-1} \cdot \mathrm{kg}^{-1} \cdot \mathrm{y}^{-1} ;{ }^{* *)}$ for the background $0.01 \mathrm{keV}^{-1} \cdot \mathrm{kg}^{-1} \cdot \mathrm{y}^{-1}$.

\begin{tabular}{|c|c|c|c|c|c|c|}
\hline Experiment & Isotope & $\begin{array}{c}\text { Mass of } \\
\text { isotope, } \mathrm{kg}\end{array}$ & $\begin{array}{l}\text { Sensitivity } \\
T_{1 / 2}, \mathrm{y}\end{array}$ & $\begin{array}{l}\text { Sensitivity } \\
\left\langle m_{\nu}\right\rangle, \mathrm{meV}\end{array}$ & Status & $\begin{array}{c}\text { Start of } \\
\text { data-tacking }\end{array}$ \\
\hline CUORE $[104,105]$ & ${ }^{130} \mathrm{Te}$ & 200 & $\begin{array}{c}6.5 \cdot 10^{26 *)} \\
2.1 \cdot 10^{26 * *)}\end{array}$ & $\begin{array}{l}20-50 \\
40-90\end{array}$ & in progress & $\sim 2013$ \\
\hline GERDA $[106,107]$ & ${ }^{76} \mathrm{Ge}$ & $\begin{array}{c}40 \\
1000\end{array}$ & $\begin{array}{l}2 \cdot 10^{26} \\
6 \cdot 10^{27}\end{array}$ & $\begin{array}{l}70-200 \\
10-40\end{array}$ & $\begin{array}{c}\text { in progress } \\
\mathrm{R} \& \mathrm{D}\end{array}$ & $\begin{array}{l}\sim 2012 \\
\sim 2015\end{array}$ \\
\hline MAJORANA $[108,109]$ & ${ }^{76} \mathrm{Ge}$ & $\begin{array}{l}30-60 \\
1000\end{array}$ & $\begin{array}{c}(1-2) \cdot 10^{26} \\
6 \cdot 10^{27}\end{array}$ & $\begin{array}{l}70-200 \\
10-40\end{array}$ & $\begin{array}{c}\text { in progress } \\
\mathrm{R} \& \mathrm{D}\end{array}$ & $\begin{array}{l}\sim 2013 \\
\sim 2015\end{array}$ \\
\hline EXO $[110,111]$ & ${ }^{136} \mathrm{Xe}$ & $\begin{array}{c}200 \\
1000\end{array}$ & $\begin{array}{c}6.4 \cdot 10^{25} \\
8 \cdot 10^{26}\end{array}$ & $\begin{array}{c}100-200 \\
30-60\end{array}$ & $\begin{array}{c}\text { in progress } \\
\mathrm{R} \& \mathrm{D}\end{array}$ & $\begin{array}{l}\sim 2011 \\
\sim 2015\end{array}$ \\
\hline SuperNEMO [112-114] & ${ }^{82} \mathrm{Se}$ & $100-200$ & $(1-2) \cdot 10^{26}$ & $40-100$ & $\mathrm{R} \& \mathrm{D}$ & $\sim 2013-2015$ \\
\hline KamLAND [115] & ${ }^{136} \mathrm{Xe}$ & $\begin{array}{r}400 \\
1000\end{array}$ & $\begin{array}{c}4 \cdot 10^{26} \\
10^{27}\end{array}$ & $\begin{array}{l}40-80 \\
25-50\end{array}$ & $\begin{array}{c}\text { in progress } \\
\mathrm{R} \& \mathrm{D}\end{array}$ & $\begin{array}{c}\sim 2011 \\
\sim 2013-2015\end{array}$ \\
\hline $\mathrm{SNO}+[121]$ & ${ }^{150} \mathrm{Nd}$ & $\begin{array}{r}56 \\
500\end{array}$ & $\begin{array}{c}4.5 \cdot 10^{24} \\
3 \cdot 10^{25}\end{array}$ & $\begin{array}{l}100-300 \\
40-120\end{array}$ & $\begin{array}{c}\text { in progress } \\
\mathrm{R} \& \mathrm{D}\end{array}$ & $\begin{array}{l}\sim 2012 \\
\sim 2015\end{array}$ \\
\hline
\end{tabular}

